\title{
Volumetric Testing with Wedges for a Nonconforming Discretization of the PMCHWT Formulation
}

\author{
Ivan Sekulic, Eduard Ubeda, and Juan M. Rius \\ AntennaLab, Signal Theory and Communications Department \\ Universitat Politècnica de Catalunya, Barcelona, 08034, Spain \\ ivan.sekulic@tsc.upc.edu
}

\begin{abstract}
The monopolar-Rao-Wilton-Glisson (RWG) discretization of the Poggio-Miller-Chan-Harrington-Wu-Tsai (PMCHWT) integral equation imposes no continuity constraints in the current expansion across the edges arising from the discretization of the boundary surface. The numerical evaluation of the hypersingular kernel contributions can be carried out through the volumetric testing of the fields over a set of tetrahedral elements attached to the boundary surface of the target. This facet-based implementation becomes well-suited for the scattering analysis of composite objects or nonconformal meshes. Furthermore, improved accuracy has been observed in the analysis of moderately small sharp-edged dielectric objects and high contrasts with the proper choice of the height of the testing tetrahedral elements. In this paper, we introduce a novel monopolar-RWG discretization of the PMCHWT formulation where wedge testing elements are adopted. We show with radar cross section results that this scheme offers improved accuracy for a wider range of heights of the testing elements than the approach with tetrahedral testing.
\end{abstract}

\section{INTRODUCTION}

The discretization of the Poggio-Miller-Chan-HarringtonWu-Tsai (PMCHWT) formulation by the method of moments [1] usually relies on the Rao-Wilton-Glisson (RWG) set, an example of divergence-conforming set, which imposes normal continuity of the currents across the edges arising from the triangulation of the boundary surface of the target. The edgebased RWG-discretization of the PMCHWT formulation is in general advantageous since the hypersingular kernel contributions appearing in the expansion of the scalar potentials, electric and magnetic, are cancelled out. However, in the analysis of composite objects, special basis functions need to be assigned to junction-edges, where several regions with different electrical properties intersect, in order to impose the proper continuity requirements [2]. Moreover, the analysis of targets meshed with nonconformal meshes, arising from the interconnection of triangulations with nonmatching edges, cannot even be considered. These pitfalls are circumvented through the development of a facet-based scheme, such as the monopolar-RWG discretization of the PMCHWT formulation, which is nonconforming because no interelement continuity constraints are imposed in the expansion of the currents [3]. The hypersingular kernel contributions are then handled by testing the fields over tetrahedral elements attached to the boundary surface, in the region where the fields, in accordance with the equivalence principle, must be zero [3]. Interestingly, improved radar cross section (RCS) accuracy with respect to the conventional RWG-PMCHWT implementation is observed for electrically small sharp-edged dielectric objects and high contrast [3]. In this paper, we propose a novel monopolarRWG discretization of the PMCHWT formulation, with wedge testing elements. We show with RCS results that the range of heights of the testing elements with observed improved accuracy increases considerably with the proposed wedge testing choice when compared with the tetrahedral choice.

\section{Nonconforming PMCHWT with Testing Wedges}

The facet-based monopolar-RWG set $\left\{\boldsymbol{m}_{n}\right\}=\left\{\boldsymbol{f}_{n}^{1}, \boldsymbol{f}_{n}^{2}\right\}$ is used to expand the electric and magnetic currents $\boldsymbol{J}_{i}$ and $\boldsymbol{M}_{i}$, residing on each side $(i=1,2)$ of the boundary surface of dielectric target as

$$
\begin{gathered}
\boldsymbol{J}_{i} \approx \sum_{n=1}^{2 N_{e}} J_{n}^{i} \boldsymbol{m}_{n}=\sum_{n=1}^{N_{e}} a_{n}^{1, i} \boldsymbol{f}_{n}^{1}+\sum_{n=1}^{N_{e}} a_{n}^{2, i} \boldsymbol{f}_{n}^{2} \\
\boldsymbol{M}_{i} \approx \sum_{n=1}^{2 N_{e}} M_{n}^{i} \boldsymbol{m}_{n}=\sum_{n=1}^{N_{e}} b_{n}^{1, i} \boldsymbol{f}_{n}^{1}+\sum_{n=1}^{N_{e}} b_{n}^{2, i} \boldsymbol{f}_{n}^{2},
\end{gathered}
$$

where $N_{e}$ denotes the number of edges arising from the triangulation of the boundary surface and the sequences $\left\{J_{n}^{i}\right\}=$ $\left\{a_{n}^{1, i}, a_{n}^{2, i}\right\},\left\{M_{n}^{i}\right\}=\left\{b_{n}^{1, i}, b_{n}^{2, i}\right\}$ represent the sets of unknown coefficients associated with the expansion of the electric and magnetic currents, respectively. The monopolar-RWG set is defined locally, on each triangle, in the same manner as the RWG set, but without imposing the normal-continuity condition across edges. The approximated scattered electric and magnetic fields in the equivalent problem associated with the region $i$ yield

$$
\begin{aligned}
\boldsymbol{E}_{i}^{s} & \approx \sum_{n=1}^{2 N_{e}} \eta_{i} \boldsymbol{T}_{n}^{i} J_{n}^{i}-\sum_{n=1}^{2 N_{e}} \boldsymbol{K}_{n}^{i} M_{n}^{i} \\
\boldsymbol{H}_{i}^{s} & \approx \sum_{n=1}^{2 N_{e}} \boldsymbol{K}_{n}^{i} J_{n}^{i}-\sum_{n=1}^{2 N_{e}} \frac{1}{\eta_{i}} \boldsymbol{H}_{n}^{i} M_{n}^{i},
\end{aligned}
$$


where the integral contributions are defined as

$$
\begin{gathered}
\boldsymbol{K}_{n}^{i}(\boldsymbol{r})=\nabla \times \iint_{\Gamma_{S}} G_{i}\left(\boldsymbol{r}, \boldsymbol{r}^{\prime}\right) \boldsymbol{m}_{n}\left(\boldsymbol{r}^{\prime}\right) d S^{\prime} \\
\boldsymbol{T}_{n}^{i}(\boldsymbol{r})=\frac{1}{j k_{i}}\left(\nabla \nabla \cdot+k_{i}\right) \iint_{\Gamma_{S}} G_{i}\left(\boldsymbol{r}, \boldsymbol{r}^{\prime}\right) \boldsymbol{m}_{n}\left(\boldsymbol{r}^{\prime}\right) d S^{\prime}
\end{gathered}
$$

and the quantities $k_{i}, \eta_{i}$, and $G_{i}$ denote the wave number, the impedance and the Green's function of the medium in region $i$, respectively. The monopolar-RWG PMCHWT is then defined on the discretized surface (S) of the dielectric object as the subtraction of the tangential components of the approximated fields in (3) and (4) [3]. The Galerkin testing of (3) and (4) cannot be undertaken because of the hypersingular kernel contributions arising in the expansion of the scalar potentials [3]. We then test the scattered fields over a set of wedges [4] attached to the boundary surface and lying in the region where, according to equivalence principle, the fields are zero (see Fig. 1). The new volumetric monopolar-RWG PMCHWT implementation with wedge testing is defined as

$$
\begin{aligned}
\iiint_{V_{m}^{2}} \boldsymbol{E}_{1}^{s} \cdot \boldsymbol{w}_{m}^{2}(\boldsymbol{r}) d V & -\iiint_{V_{m}^{2}} \boldsymbol{E}_{2}^{s} \cdot \boldsymbol{w}_{m}^{1}(\boldsymbol{r}) \\
& =-\iiint_{V_{m}^{2}} \boldsymbol{E}^{i n c} \cdot \boldsymbol{w}_{m}^{2}(\boldsymbol{r}) d V
\end{aligned}
$$

where $m=1,2, \ldots, 2 N_{e}$. The target is immersed in free space (region 1), where the incident electric field $\boldsymbol{E}^{\text {inc }}$ propagate. The corresponding magnetic-field integral equation replaces incident electric field $\boldsymbol{E}^{i n c}$ with incident magnetic field $\boldsymbol{H}^{i n c}$. The $m$ th testing wedge attached to the boundary surface is denoted by $V_{m}^{i}$ in region $i$, where where the wedge testing functions $\left\{\boldsymbol{w}_{m}^{i}\right\}$ are defined as in [4] (see Fig. 1). Also, it is assumed that $J_{n}^{1}=-J_{n}^{2}$ and $M_{n}^{1}=-M_{n}^{2}$ when constructing the matrix system in (7).

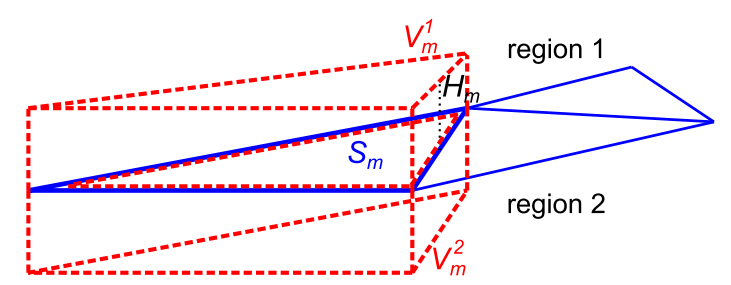

Fig. 1. Pair of volumetric wedge elements, $V_{m}^{1}$ and $V_{m}^{2}$, attached to the triangular facet $S_{m}$, and defined, respectively, inside regions 1 and 2 .

In an analogous fashion as the volumetric PMCHWT implementation with tetrahedral testing elements [3], the accuracy of this implementation is fine-tuned by varying the height of the wedge elements $(H)$, which we define, in view of Fig. 1, with the same value in both regions, as a fraction of $h$, the average side length of the corresponding surface triangle.

\section{NUMERICAL RESULTS}

In Fig. 2, we illustrate for an electrically-small dielectric cube with side $0.1 \mathrm{~m}$ and high contrast $\left(\epsilon_{r}=100\right)$, the RCS errors computed with the monopolar-RWG discretizations of the PMCHWT with wedge testing, noted as monoRWG-wed, or with tetrahedral testing, noted as monoRWG-tet, with respect to the RWG-discretization, noted as RWG. We compute the relative root-mean-square RCS errors for a wide range of testing heights $H$ over 60 directions in the $y-z$ scattering plane. The relative errors are referred to RCS reference computed with PMCHWT and very fine degree of meshing yielding 32,400 unknowns. In order to establish a fair comparison, the involved formulations handle similar number of unknowns, but different number of edges $N_{e}$ since the monopolar-RWG implementations define two unknowns per edge. The target is impinged by an $x$-polarized $+z$-propagating plane wave, the free-space wavelength $\lambda_{0}$ is set to $1 \mathrm{~m}$, and all the testing elements are defined conformal to the boundary [4]. It is clear that wedge testing shows improved accuracy with respect to RWG for a wider range of testing heights $H(h / 30>H>$ $\left.h / 10^{4}\right)$ than tetrahedral testing $(h / 2>H>h / 10)$ (see Fig. 2).

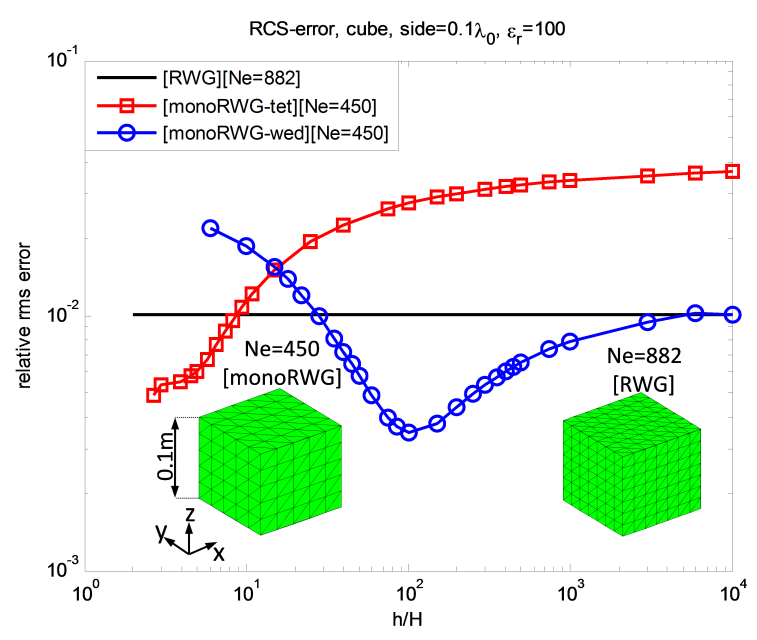

Fig. 2. Relative rms RCS-errors of the volumetric monopolar-RWG PMCHWT implementations versus the height of the testing entities $H$ of a dielectric cube $\left(\epsilon_{r}=100\right)$ with side $0.1 \lambda_{0}$ under an impigning $x$-polarized $+z$-propagating plane wave, $\lambda_{0}=1 \mathrm{~m}$.

\section{ACKNOWLEDGMENT}

This work was supported by the Spanish "Comisión Interministerial de Ciencia y Tecnología" (CICYT) under projects TEC2013-47360-C3-1-P and TEC2016-78028-C3-1-P.

\section{REFERENCES}

[1] A. J. Poggio and E. K. Miller, "Integral equation solutions of threedimensional scattering problems," in Computer Techniques for Electromagnetics, R. Mittra, Ed. Oxford, UK: Pergamon Press, 1973.

[2] Branko M. Kolundzija, "Electromagnetic modeling of composite metallic and dielectric structures," IEEE Trans. Microw. Theory Tech, vol. 47, no. 7, July 1999.

[3] I. Sekulic, E. Ubeda, and J. M. Rius, "Nonconforming discretization of the PMCHWT integral equation applied to arbitrarily shaped dielectric objects," European Conference on Antennas and Propagation (EuCAP), Mar. 2017, Paris, France.

[4] E. Ubeda, Juan M. Rius, A. Heldring, and I. Sekulic, "Volumetric testing parallel to the boundary surface for a nonconforming discretization of the electric-field integral equation," IEEE Trans. Antennas Propag., vol. 63 , no. 7, pp. 3286-3291, July 2015. 